\title{
Ancestry Estimation in South Africa Using Craniometrics and Geometric Morphometrics
}

\author{
Kyra E. Stull, $\mathrm{PhD}^{1}$; Michael W. Kenyhercz, $\mathrm{MS}^{2,3}$; Ericka N. L’Abbé, $\mathrm{PhD}^{4}$ \\ ${ }^{1}$ Department of Anthropology, Idaho State University \\ 921 South $8^{\text {th }}$ Ave, Stop 8005, Pocatello, ID USA 83209 \\ kstullster@gmail.com \\ ${ }^{2}$ Department of Anthropology, 303 Tanana Loop, Bunnell Building Room 405A, University of Alaska \\ Fairbanks, Fairbanks, AK USA 99775 \\ michael.kenyhercz@gmail.com \\ ${ }^{3}$ Department of Anthropology, University of Tennessee, Knoxville, 250 South Stadium Hall, Knoxville, \\ Tennessee USA 37996 \\ ${ }^{4}$ Department of Anatomy, Faculty of Health Sciences, University of Pretoria, Private Bag x323, Arcadia, \\ South Africa 0007 \\ ericka.labbe@up.ac.za
}

Running Title: Ancestry Estimation in South Africa

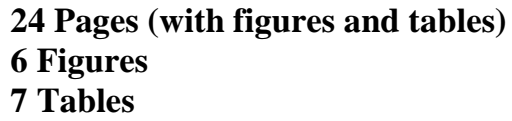

Corresponding Author:

Kyra Stull

Department of Anthropology

Idaho State University

921 South $8^{\text {th }}$ Ave, Stop 8005

Pocatello, ID USA 83209

Phone: +1 (864) 230-2301

Email: kstullster@gmail.com

\begin{abstract}
Population history and positive assortative mating directs gene flow in such a way that biological differences are recognized among groups. In turn, forensic anthropologists quantify biological differences to estimate ancestry. Some anthropologists argue that highly admixed population groups, such as South African coloureds, cannot achieve acceptable accuracies because within group variance is too large. Whereas ancestry estimation in South Africa has been limited to craniometric data from South African
\end{abstract}


blacks and whites, the current study integrates craniometric and geometric morphometric data from the three largest South African groups.

Crania from 377 South African individuals (black $=158$, white $=112$, and coloured $=107)$ comprised the sample. Standard measurements were collected and the coordinate data were subjected to Generalized Procrustes Analysis (GPA), which resulted in size-free shape variables (ProCoords). A principal component analysis was used to combine the shape variation captured in the ProCoords (ProCoords PC). Linear discriminant analysis (LDA), using equal priors, stepwise variable selection and leaveone-out cross-validation, was conducted on the ProCoords, the ProCoords PCs, and the traditional craniometric data.

The LDA using 18 stepwise selected ProCoords resulted in the highest crossvalidated accuracy (89\%). Utilization of geometric morphometric data emphasized that the relative location of cranial landmarks was more discriminating than simple linear distances. Regardless of high levels of genetic admixture, South African coloureds are a homogeneous group and morphologically distinct from other contemporaneous South African populations. Furthermore, the present study demonstrated a correspondence between peer-reported race and morphological differences in the crania of black, white, and coloured South Africans.

Keywords: Generalized Procrustes analysis; linear discriminant analysis; craniometric variation; human variation 


\section{Introduction}

Throughout much of the world, social race remains a reality in which persons attribute themselves to different groups, either socially, or, in the case of the population census, legally. Social race is a concept of identification based on cultural, historical or familial affiliation and has no biological reality [1-4]. However, morphological differences are recognized among groups because positive assortative mating, geographical distances, and social forces act as barriers for gene flow, thus limiting group interaction and increasing the variation between groups [5-7]. In turn, forensic anthropologists quantify morphology to estimate ancestry, or social race, which provides practical information that is useful in a medico-legal setting $[2,5]$.

From 1948 to 1990, South Africans were forcibly separated according to race in public, geographic locality, and education. In South Africa, the former racial classification system is maintained within all bureaucratic systems in the country but now the terms are based on self-perception and self-identification [8-10]. Institutionalized racism drastically affected gene flow in modern South Africa. However, colonization and migration shaped the unique constructs of the South African population prior to the implementation of various segregation laws and is thus considered the foundation to modern variation in South African groups.

In 1652, the Dutch East India Company established a fueling station in what is known today as Cape Town, South Africa. After some time, Europeans, primarily Dutch with a scattering of French Huguenots and Germans, began to create permanent settlements in the area $[8,11]$. A large number of English settlers followed in the mid1800s. In order to maintain the rapid expansion of Cape Town, slaves were introduced 
from Central Africa, Madagascar, India and Indonesia, among other countries, as well as from indigenous populations of southern Africa, such as the Khoikhoi and San [11,12]. Historical records indicated marriage among white males and free black and indigenous females was common $[8,13]$. During this time in history, religion influenced status more than skin color, such that the social status of black or indigenous women would increase if they were considered Christian, which often led to marriage and miscegenation [14,15]. Acceptance of children from cross-cultural relations ranged from full acceptance to slave status. However, the fluidity surrounding race relations decreased in the early 1800's [15]. Inter-racial relations became social taboo and the frequency of mixed marriages decreased and were eventually outlawed with the Prohibition of Mixed Marriages Act of 1949 [13].

Even prior to the arrival of the Europeans, admixture occurred among the indigenous groups, specifically among the Khoikhoi and San (commonly pooled and referred to as Khoe-San), and Bantu-speakers [16,17]. Nine of the 11 South African national languages are recognized as Bantu languages, a sub-group of the Niger-Congo languages. However, one of the South African Bantu languages, isiXhosa, contains clicks similar to those found within the Khoisan linguistic group that is associated with the Khoe-San. The presence of Khoisan characteristics in a Bantu language and the archaeological evidence of shared technologies suggest gene flow between Bantuspeakers and Khoe-San. Even though gene flow is evident between Bantu-speakers and Khoe-San, the groups are considered distinct in South Africa [11,18]. In sum, considerably complex interactions of different peoples and cultures form the foundation of modern South Africa. 
According to the 2011 South African census, the three largest groups in South Africa are blacks (79\%), whites (9\%), and coloureds (9\%) [10]. The remainder of the population consists of Indians and Asians. Individuals who were a product of the Bantu expansion and who were not Khoe-San, were grouped into a single entity and considered black South Africans underneath the apartheid government [13]. Thus, contemporary black South Africans are comprised of individuals from numerous ethnic groups that largely self-identify with language. Coloureds are a self-identified group unique to South Africa $[8,11]$, whose history and genetic admixture are often relayed through documents on slavery, marriage, and personal accounts of the settlement of Cape Town in the $17^{\text {th }}$ and $18^{\text {th }}$ centuries. The term coloured dates back to 1808 , following the abolishment of slavery $[8,12]$. The distinct group, which emerged as a result of this complex history, displays the highest levels of intra- and inter-continental genetic admixture compared to all other populations in the world $[11,19,20]$. On average, the genetic composition of coloureds is an equal contribution from four groups, namely European, Bantu-speakers, Khoe-San and Indian [19-21]; however, genetic contributions vary between the sexes, at the individual level, and in geographic location within South Africa [22].

Historical circumstances contribute to cultural and social behavior, which subsequently modifies the range of human variation within a population. Specifically, positive assortative mating alters trait frequencies and biologically modifies social groups. Although the genetic structure of South Africa's populations demonstrates past admixture, institutionalized racism and positive assortative mating has left these groups largely segregated from one another and subsequently has decreased variation within groups and increased variation between groups. South African whites, blacks, coloureds and Asians 
all have high rates (> 96\%) of homogamy, even after the termination of institutionalized racism [13]. Though apartheid is likely the most prominent force driving separation among South Africans, other cultural barriers, such as the polylinguistic society, reduces the rate of heterogamy [13]. Thus, South Africa's history, and specifically South Africa's race history, can be used to identify differences among groups [23-25]. Similarly, race relations were used to interpret modern craniometric variation among black and white North Americans. In North America, a strong concordance exists between cranial morphology and social race, seen by correct classifications as high as $97 \%[5,26]$.

Yet, some anthropologists assume that high levels of genetic admixture, as found amongst coloured South Africans, will present with such a wide spectrum of variation that attempts to estimate ancestry will fail and the unidentified remains will be meaninglessly cast into white and non-white divisions [27,28]. Theoretically, this abovementioned assumption is flawed because panmixia does not exist; as described earlier, cultural, social, and legal barriers have restricted gene flow among groups in South Africa and elsewhere. Previous studies using modern South African crania have been limited to standard craniometric analyses between black and white South Africans. When modern South African craniometric data were explored by group and sex, accuracies of $71 \%$ were achieved [24], but when mid-facial variables were explored by group only, accuracies increased to 95\% [23]. The purpose of this study was to estimate ancestry and to evaluate craniometric patterns in the three largest social groups of South Africa through craniometric and geometric morphometric techniques. Because craniometric data reflects genetic relationships, multivariate statistical analyses should reveal three largely unique social groups $[5,7,29,30]$. 


\section{Materials and Methods}

A total of 377 crania were used in the analyses and included South African (SA) black $(\mathrm{n}=158)$, white $(\mathrm{n}=112)$, and coloured $(\mathrm{n}=107)$ individuals. Because the same landmarks were not available on every cranium, the sample sizes for the geometric morphometric analyses were reduced to a total of 209 individuals $($ black $=101$, white $=$ 58 , and coloured $=50$ ). The skeletal remains were housed in different skeletal collections in South Africa, including the Pretoria Bone Collection at the University of Pretoria, the Raymond A. Dart Collection of Human Skeletons at the University of Witwatersrand, and the Kirsten Skeletal Collection at the University of Stellenbosch. Although males and females comprised the sample, the sexes were pooled for all analyses because the aim was to explore population differences. All individuals were older than 18 years of age and did not exhibit signs of pathology, traumatic injuries or extensive antemortem tooth loss.

Coordinate data were collected with a three-dimensional digitizer and 3Skull [31] . In an effort to retain large sample sizes, 44 cranial landmarks were chosen to represent the entire cranium with special reference to the mid-face, which previously achieved high accuracies distinguishing modern South African groups (Table 1) [23].

Coordinate data were subjected to Generalized Procrustes Analysis (GPA) in the program MorphoJ [32]. A GPA is a superimposition technique that translates, scales, and rotates the landmark combinations upon the centroid size and location for each specimen [33]. Because GPA minimizes the squared difference between homologous landmarks, the resultant Procrustes coordinates (ProCoords) are size-free shape variables. A principal 


\begin{tabular}{cc|cc}
\hline \multicolumn{4}{l}{ Table 1. Cranial landmarks used in the current study. } \\
\hline Number & Landmark & Number & Landmark \\
\hline 1 & Alare L & 23 & Jugale R \\
2 & Alare R & 24 & Lambda \\
3 & Alveolon & 25 & Mastoideale L \\
4 & Asterion L & 26 & Mastoideale R \\
5 & Asterion R & 27 & Nasion \\
6 & Radiculare L (zygomatic root) & 28 & Most Inferior Nasal Border L \\
7 & Radiculare R & 29 & Most Inferior Nasal Border R \\
8 & Basion & 30 & Orbital Height Inferior Point \\
9 & Bregma & 31 & Orbital Height Superior Point \\
10 & Dacryon L & 32 & Opisthocranion \\
11 & Dacryon R & 33 & Opisthion \\
12 & Ectoconchion L & 34 & Porion L \\
13 & Ectoconchion R & 35 & Porion R \\
14 & Eurion L & 36 & Prosthion \\
15 & Eurion R & 37 & Subspinale \\
16 & Frontomalare Anterior L & 38 & Staurion \\
17 & Frontomalare Anterior R & 39 & Frontotemporale L (wfbl) \\
18 & Foramen Magnum Breadth L & 40 & Frontotemporale R \\
19 & Foramen Magnum Breadth R & 41 & Maximum Frontal Point L \\
20 & Glabella & 42 & Maximum Frontal Point R \\
21 & Hormion & 43 & Zygomaxilare L \\
22 & Jugale L & 44 & Zygomaxilare R \\
\hline & & &
\end{tabular}

ProCoords for the entire cranium. The PCA of the ProCoords (ProCoords PCs) yielded 125 principal components $(\mathrm{PC})$ using the variance-covariance matrix. The average of the PC variances was used as a cut-off score, as limiting the number of components based on a PC contributing an eigenvalue greater than one is not practical [34]. Thus, only $30 \mathrm{PCs}$ from the original 125 PCs were used for the analyses.

One strength of geometric morphometrics is the graphical presentation of shape changes, both in direction and in magnitude of change [35]. Thus, the ProCoords PCs 
were plotted to demonstrate the shape changes among the crania. The resultant graphs generated through MorphoJ are known as lollipop graphs. The lollipop "circle" represents the mean starting shape and the stem shows, incrementally, the positive shape changes in principal component units to the target shape [23]. Essentially, the longer the stem, the greater the magnitude of shape change in that particular landmark.

The craniometric data, ProCoords, and ProCoords PCs were subjected to linear discriminant analysis (LDA), which is a linear combination of measurements used to assign group membership. The LDA explored group differences, thus the sexes were pooled for each analysis. Equal prior probabilities were used because sample sizes varied among the three groups. Additionally, the sample size among all groups was larger than three times the number of measurements $(3 \mathrm{~m})$ included for each model creation to ensure the model was not overfit [36]. Each LDA model utilized forward stepwise variable selection to choose a subset of the variables that were the most useful for group separation. Lastly, a leave-one-out cross-validation (LOOCV) procedure was used, wherein one specimen is removed from the sample and used to test the discriminant function that was created based on the remaining specimens. The LOOCV process reduces bias in the estimates and supplies a realistic estimate of prediction error because the error is averaged from the results of the held-out specimens [37]. The Mahalanobis distance $\left(D^{2}\right)$ matrix is presented with each discriminant analysis as a means to demonstrate the overall similarity among groups [38]. 


\section{Results}

Generalized Procrustes Analysis

Principal component 1 accounted for $17.9 \%$ of the total shape variation. The major movement in PC1 was with landmarks 14 and 15 (left and right euryon, respectively), showing a tendency toward a mostly inferior and slightly anterior migration and, to a lesser degree, opisthocranion (landmark 32) moving antero-superiorly (Figure 1).

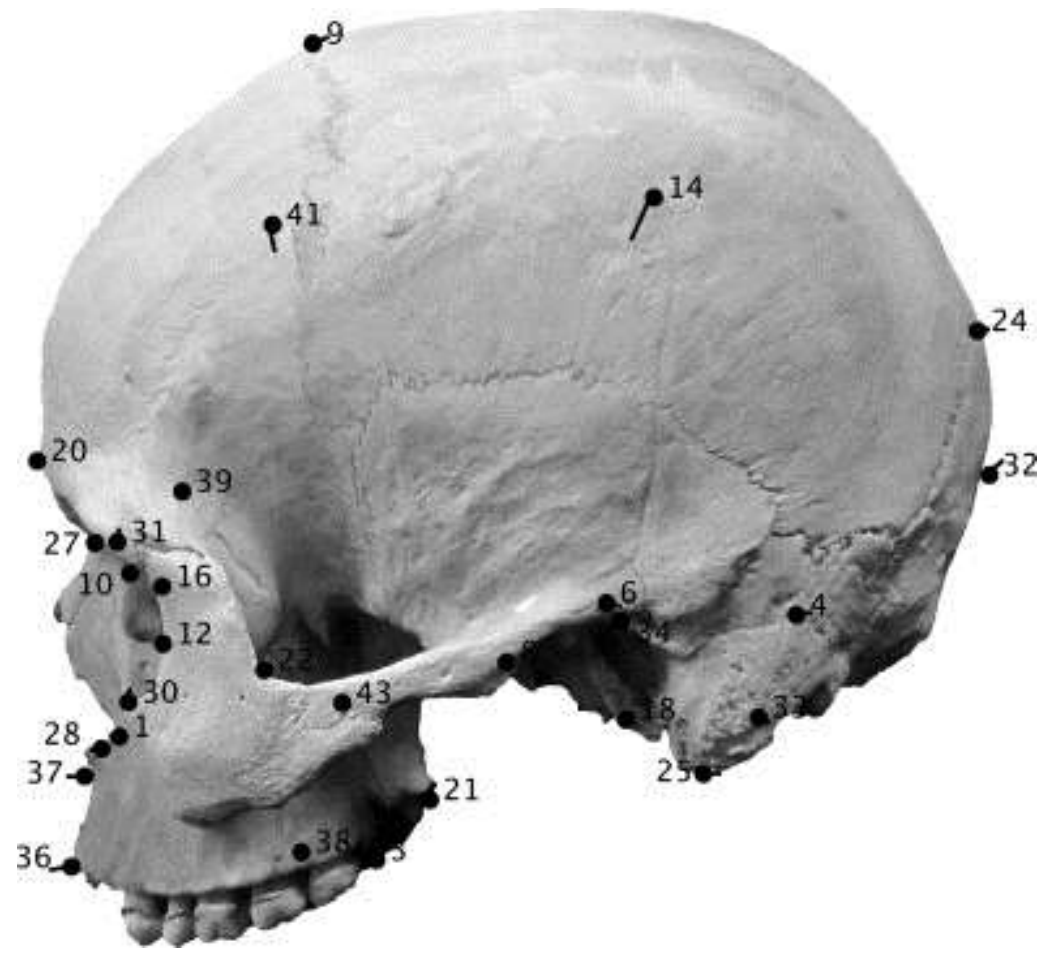

Fig. 1 : Lollipop graph superimposed on lateral view of cranium showing the shape changes associated with PC1. Landmarks collected on the right side of the cranium were excluded in the figure for ease of viewing; please refer to Table 1 for the landmarks associated with each number.

\section{Linear Discriminant Analysis}

The LDA using the traditional craniometrics resulted in an overall cross-validated correct classification rate of $84 \%$ using 11 stepwise selected variables (Table 2). South 


\begin{tabular}{|c|c|c|c|c|c|}
\hline & \multirow{2}{*}{ Total Number } & \multicolumn{3}{|c|}{ Into Group } & \multirow{2}{*}{$\begin{array}{l}\text { Percent } \\
\text { Correct }\end{array}$} \\
\hline & & Black & Coloured & White & \\
\hline Black & 158 & 143 & 8 & 7 & $91 \%$ \\
\hline Coloured & 107 & 3 & 94 & 10 & $88 \%$ \\
\hline White & 112 & 9 & 14 & 89 & $80 \%$ \\
\hline \multicolumn{6}{|c|}{ Total Correct: 326 out of 377 (84\%) Cross-validated } \\
\hline
\end{tabular}

African blacks achieved the highest accuracy (91\%) and SA whites achieved the lowest accuracy (80\%). South African whites and coloureds misclassified more frequently as one another, than either misclassified as SA black. The increased similarity between SA coloureds and SA whites is demonstrated by the smaller $D^{2}$ values (Table 3 and Figure 2).

\begin{tabular}{cccc}
\hline \multicolumn{3}{l}{ Table 3. Mahalanobis $\mathrm{D}^{2}$ for the traditional craniometrics. All } \\
distances are significant at $\mathbf{p}<\mathbf{0 . 0 0 1}$. & Coloured & White \\
\hline & Black & & \\
Black & - & - & \\
Coloured & 12.42 & 7.71 & - \\
White & 14.96 & \\
\hline
\end{tabular}




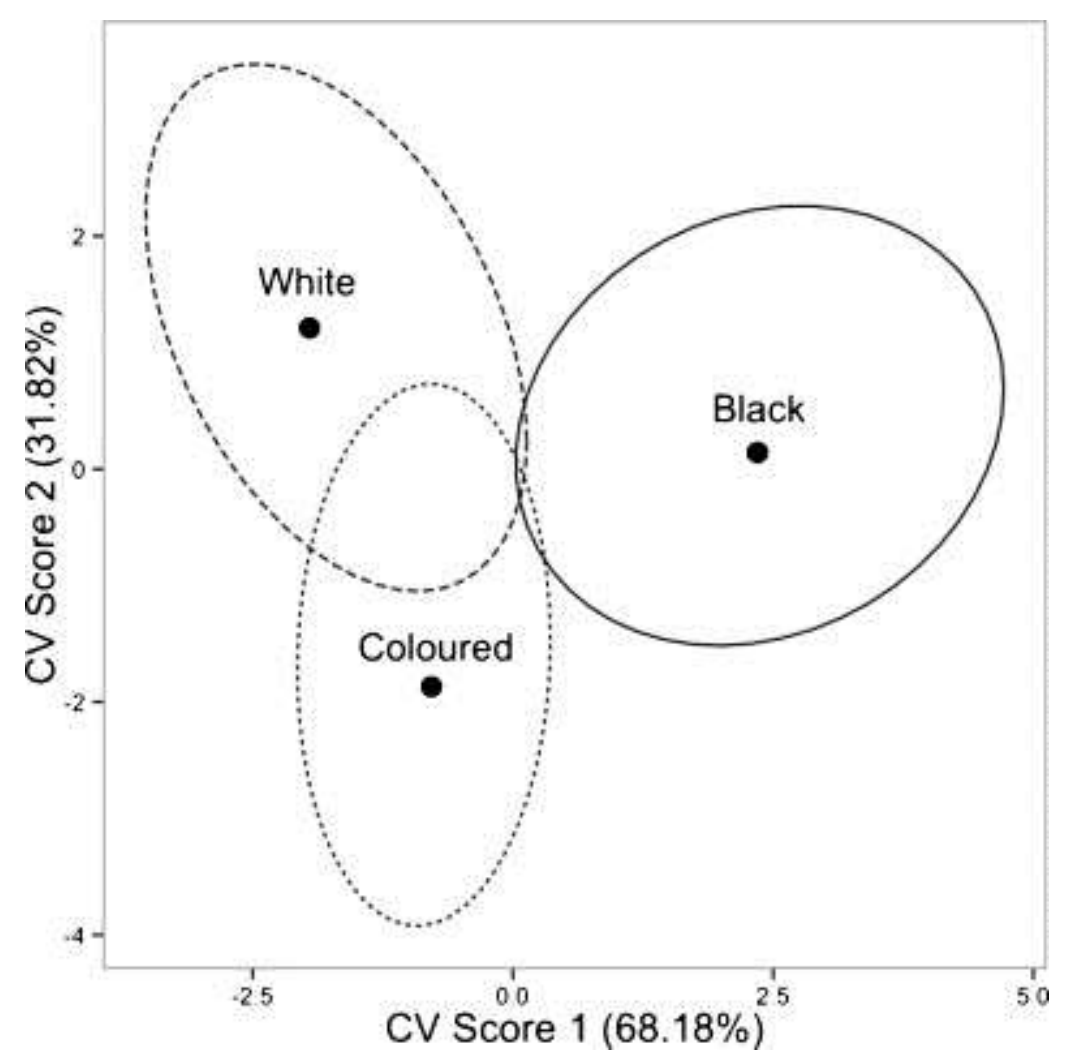

Fig. 2 : Plot of canonical variate group means and 95\% confidence ellipses for the LDA using traditional craniometrics. Overall cross-validated accuracy was $87 \%$. Please refer to Table 2 for group abbreviations.

\begin{tabular}{cc|ccc|c}
\hline \multicolumn{7}{c}{ Table 4. LDA results using 18 Forward Wilks selected ProCoords. } \\
\hline \multirow{3}{*}{ Total Number } & \multicolumn{4}{c}{ Into Group } & Percent \\
\cline { 3 - 6 } Black & 101 & Black & Coloured & White & Correct \\
\cline { 2 - 6 } Coloured & 50 & 51 & 9 & 1 & $90 \%$ \\
White & 58 & 0 & 41 & 4 & $82 \%$ \\
\hline \multicolumn{5}{r}{ Total Correct: 186 out of 209 (89\%) Cross-validated } \\
\hline
\end{tabular}

Using 18 stepwise selected ProCoords, LDA resulted in a cross-validated total correct classification of $89 \%$ (Table 4). The individual ProCoords stepwise selected focused on midface variables (i.e., the $y$-coordinate for subspinale had the largest 
coefficient in the first discriminant function). In contrast to the craniometric LDA, SA whites achieved the highest accuracies (93\%) and SA coloureds achieved the lowest accuracies (82\%). The $D^{2}$ matrix demonstrated the increased similarity between SA coloureds and SA blacks compared to SA coloureds and SA whites (Table 5 and Figure $3)$.

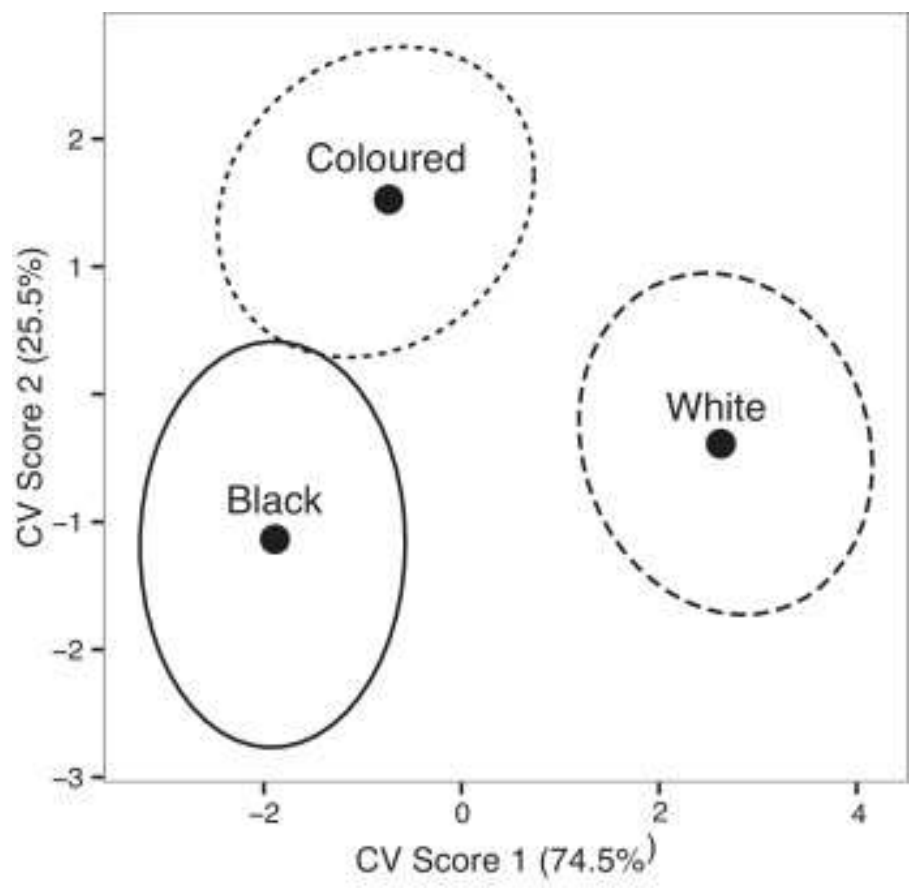

Fig. 3 : Plot of canonical variate group means and 95\% confidence ellipses for the LDA using ProCoords. Overall cross-validated accuracy was 89\%; please refer to Table 2 for group abbreviations.

\begin{tabular}{cccc}
\hline \multicolumn{4}{l}{ Table 5. Mahalanobis $\mathbf{D}^{2}$ matrix for ProCoords. All distances } \\
are significant at $\mathbf{p}<\mathbf{0 . 0 0 1}$. & & \\
\hline & Black & Coloured & White \\
Black & - & & \\
Coloured & 8.39 & - & - \\
White & 20.93 & 14.96 & - \\
\hline
\end{tabular}




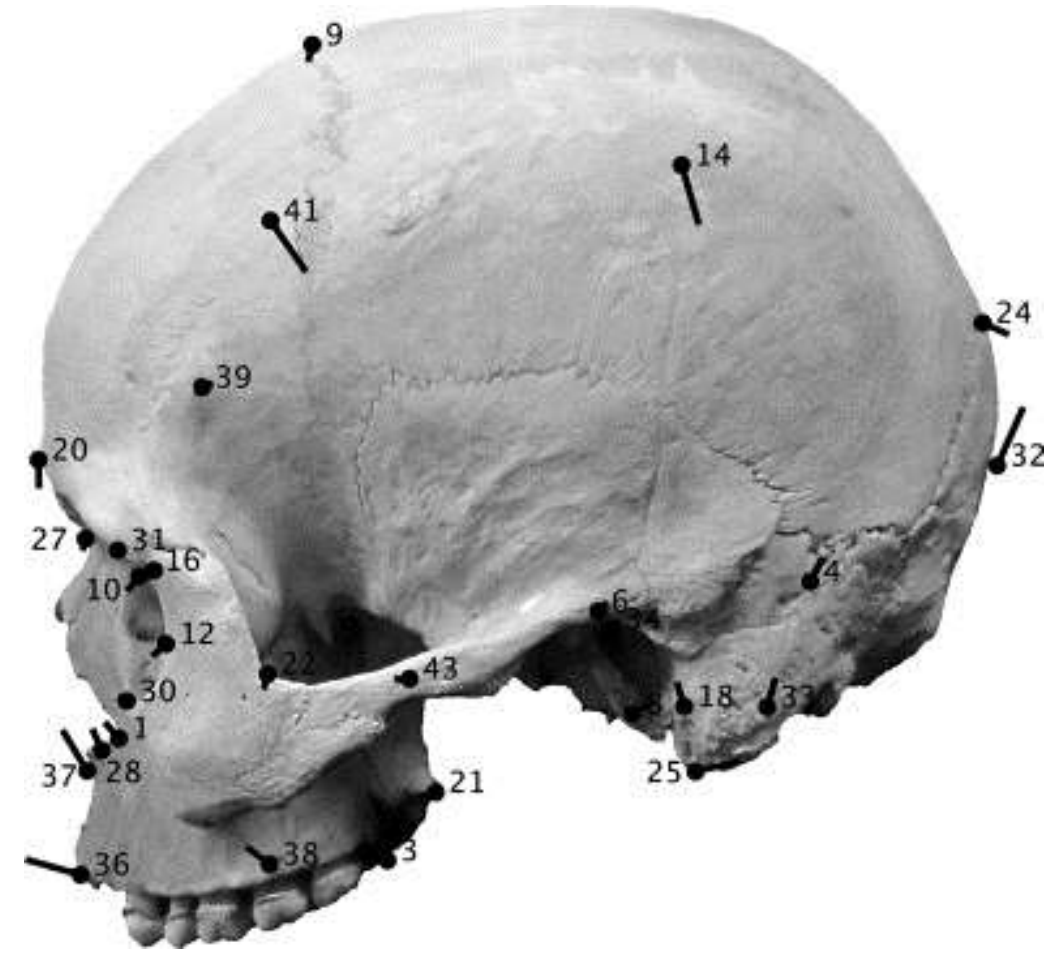

Fig. 4 : Lateral view of the shape changes associated with CV1. Landmarks collected on the right side of the cranium were excluded in the figure for ease of viewing; please refer to Table 1 for the list of cranial landmarks.

Canonical variate (CV) one accounted for $74.5 \%$ of the variation and indicated major shape differences between white and black South Africans (Figure 4). South African blacks demonstrated the greatest negative mean $\mathrm{CV}$ score, followed by SA coloureds, while SA whites had a positive CV mean. Major shape changes involved the inferior placement of left and right euryon (landmarks 14 and 15) in SA whites compared to SA blacks and coloureds (Figure 4). Both euryon landmarks were also more medially placed in SA whites as compared to the other groups. South African whites demonstrated a more inferior placement of minimum frontal breadth and, similar to placement of euryon in SA whites, the minimal frontal breadth was also more medially oriented than black and coloured groups. Canonical variate two accounted for the remaining $25.5 \%$ of 
the variation and mostly demonstrated differences between SA coloureds and the other two groups. South African coloureds demonstrated the greatest positive mean CV2 score while SA blacks demonstrated the greatest negative mean score. The major shape changes in CV2 were with euryon, which was more supero-posteriorly positioned in SA coloureds compared to SA blacks and whites (Figure 5).

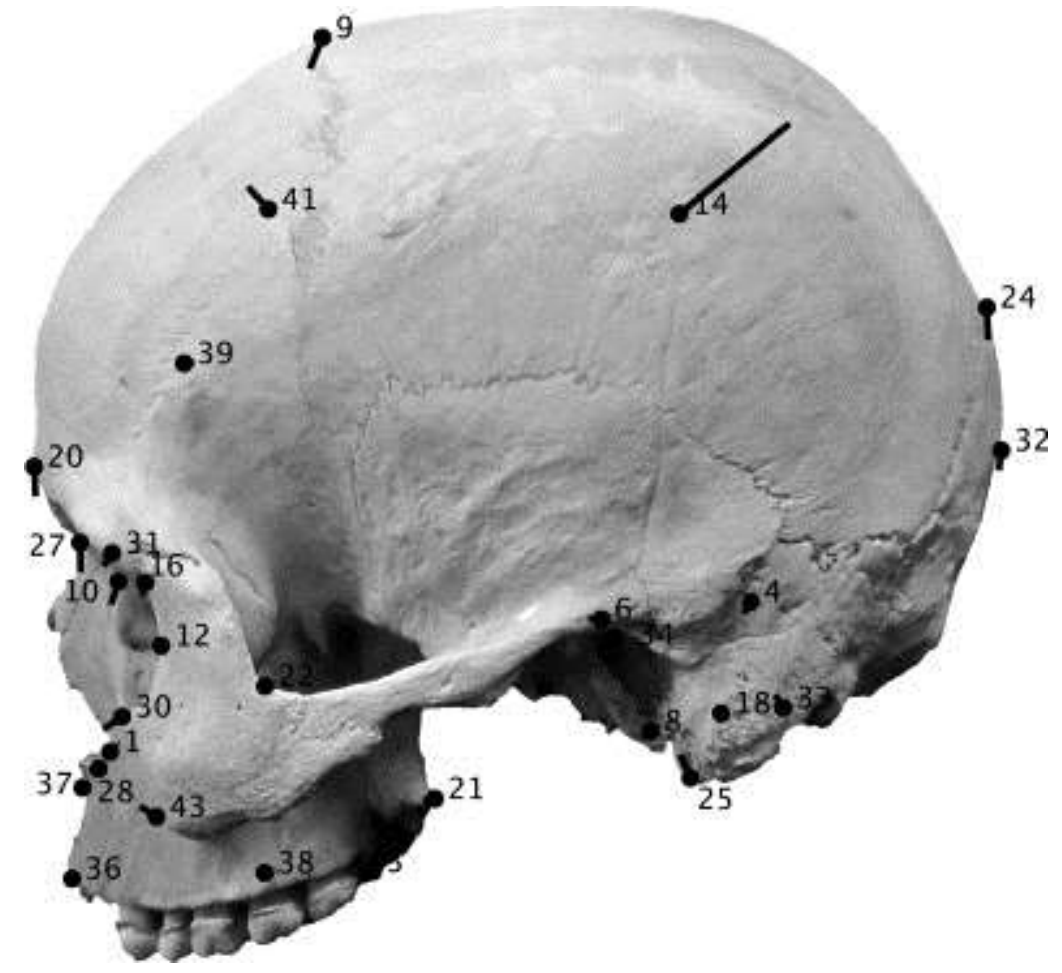

Fig. 5 : Lateral view of the shape changes superimposed on a cranium associated with CV2. Landmarks collected on the right side of the cranium were excluded in the figure for ease of viewing; please refer to Table 1 for the list of cranial landmarks.

\begin{tabular}{|c|c|c|c|c|c|}
\hline & \multirow{2}{*}{$\begin{array}{c}\text { Total } \\
\text { Number }\end{array}$} & \multicolumn{3}{|c|}{ Into Group } & \multirow{2}{*}{$\begin{array}{l}\text { Percent } \\
\text { Correct }\end{array}$} \\
\hline & & Black & Coloured & White & \\
\hline Black & 101 & 79 & 18 & 4 & $78 \%$ \\
\hline Coloured & 50 & 10 & 33 & 7 & $66 \%$ \\
\hline White & 58 & 4 & 1 & 53 & $91 \%$ \\
\hline
\end{tabular}


The LDA of the ProCoords PCs resulted in a 79\% total correct classification using 18 stepwise selected variables (Table 6). South African blacks mainly misclassified as SA coloureds, and SA coloureds misclassified relatively evenly as black or white South Africans. However, in contrast to previous analyses, SA whites misclassified more as SA blacks than SA coloureds. The $D^{2}$ matrix demonstrated more similarity between SA blacks and coloureds compared to SA whites and coloureds (Table 7 and Figure 6).

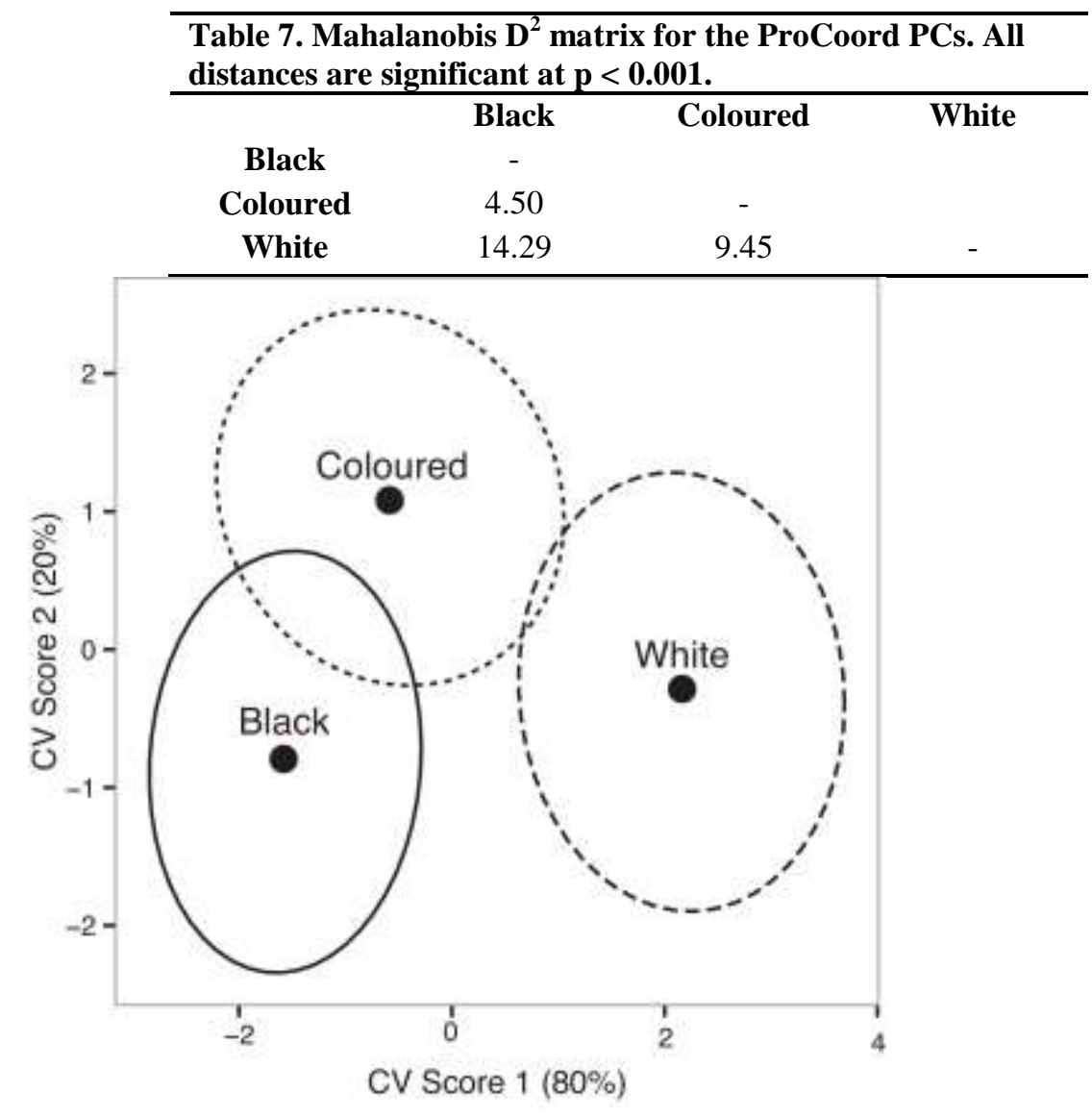

Fig. 6 : Plot of canonical variate group means and 95\% confidence ellipses for the LDA using ProCoords PCs. Overall cross-validated accuracy was 79\%; please refer to Table 2 for group abbreviations. 


\section{Discussion}

A strong correlation between cranial morphology and genetics has consistently been demonstrated in the literature [7,39-41]. Additionally, Tang et al. [42] presented a strong agreement $(99.86 \%)$ between the major genetic groups in the United States and the self-identified social group of each individual. Similarly, Ousley et al. [5] demonstrated a high concordance between social race and morphological differences in the crania of American blacks and whites. In the present study, classification accuracies reached $89 \%$ using the ProCoords in a three-way LDA, which is much greater than chance and offers practical applicability for forensic anthropologists. The present study demonstrated a correspondence between peer-reported race and cranial morphology of blacks, whites, and coloured South Africans and refuted the claim that highly admixed populations cannot achieve high accuracy rates. Although colonization in the 1600's created an extremely heterogeneous population in South Africa, positive assortative mating and institutionalized racism resulted in high rates of homogamy within the socially constructed South African groups. Simply, coloured South Africans display the highest level of genetic admixture, but the population is distinct from contemporaneous South African groups.

The ProCoords maximized group separation better than the linear craniometrics or ProCoords PCs. Additional researchers also concluded that shape variables resulted in higher classification rates compared to linear measures [43]. Because the ProCoords and ProCoords PCs capture different aspects of cranial variation, their capabilities as discriminators differ. For example, the ProCoords PCs accounted for shape changes throughout the entire cranium as opposed to the measurements (coordinates) that varied 
the most. Some regions of the crania, such as the basal region, showed great overlap among groups and the inclusion of these variables in the ProCoords PCs likely resulted in redundant information and, thus, an overall lower discriminatory power. The higher accuracy of the ProCoords than the linear measures indicates that the relative location of landmarks is perhaps more important than simple linear distances and that shape data can better elucidate the relationships between populations that occupy the same geographic space in South Africa. For example, maximum cranial breadth was not recognized as an important variable using linear measures but the relative placements of left and right euryon had great discriminating power, as depicted in Figures 4 and 5. The authors acknowledge that euryon is considered a Type II landmark, which would inherently incur more measurement error; however, the relative position indicated trends as SA whites had a more inferiorly placed euryon in comparison to SA blacks and coloureds (Figure 4) and SA coloureds had a much more posteriorly placed euryon as compared to SA blacks and whites (Figure 5). In the pursuit of classification, the overarching criterion should be enhanced model performance as judged by total correct classifications.

As demonstrated by the $D^{2}$ matrices, SA coloureds are more similar in size to white South Africans, via the craniometric analysis, but are more similar in shape to black South Africans, via both geometric morphometric analyses. Although the present study was solely interested in large-scale population differences, the effects of sex need to be considered and how those affect size and shape differences within each population, specifically in SA coloureds.

While social race, and even peer-reported race, has no meaningful or direct relationship with biology and is often referred to as the "product of scientific imagination" 
(page 3) [44], forced segregation around race and cultural attributes "plays a role in clinal models of genetic variability" (page 31) [45]. The most likely cause of differing trait frequencies in the South African population is that of cultural constraints and how these affect mate selection [45]. Colonialism, slavery, segregation, as well as political and cultural barriers shaped human variation in South Africa.

\section{Conclusions}

Despite the high levels of admixture evident in the South African populations, especially in SA coloureds, linear discriminant analyses using craniometrics and geometric morphometrics were able to identify group differences with high crossvalidated accuracies (89\%). Geometric morphometrics outperformed traditional craniometrics and the PCA of shape variables in estimating ancestry of unknown persons in South Africa. Several hundred years of migration, colonialism and forced separation resulted in a strong correspondence between social identity in South Africa and skeletal morphology. The present study is the first to evaluate both cranial shape and size differences among the three largest South African groups, which offers proof that ancestry estimation is possible even in highly admixed populations and ultimately aids in a better understanding of craniometric and morphometric variation in modern South African groups.

\section{References}

[1] C. Brace, A Four-Letter Word Called "Race," in: L.T.R. and L. Lieberman (Ed.), Race and Other Misadventures: Essays in Honor of Ashley Montagu in His Ninetieth Year, General Hall, Inc., Dix Hills, NY, 1996: pp. 106-141.

[2] N.J. Sauer, Forensic anthropology and the concept of race: If races don't exist, why are forensic anthropologists so good at identifying them?, Soc Sci Med. 34 (1992) 107-111. 
[3] R. Caspari, Deconstructing Race: Racial Thinking, Geographic Variation, and Implications for Biological Anthropology, in: A Companion to Biological Anthropology, Wiley-Blackwell, 2010: p. 608.

[4] I.F. Haney Lopez, The Social Construction of Race, in: R. Delgado (Ed.), Critical Race Theory 1st Ed, 1st edition, Temple University Press, Philadelphia, 1994: pp. 191-203.

[5] S. Ousley, R. Jantz, D. Freid, Understanding race and human variation: Why forensic anthropologists are good at identifying race, American Journal of Physical Anthropology. 139 (2009) 68-76.

[6] N. Risch, E. Burchard, E. Ziv, H. Tang, Categorization of humans in biomedical research: genes, race and disease, Genome Biol. 3 (2002) 1-12.

[7] J.H. Relethford, Race and global patterns of phenotypic variation, American Journal of Physical Anthropology. 139 (2009) 16-22.

[8] N. Patterson, D.C. Petersen, R.E. van der Ross, H. Sudoyo, R.H. Glashoff, S. Marzuki, et al., Genetic structure of a unique admixed population: implications for medical research, Hum. Mol. Genet. 19 (2010) 411-419. doi:10.1093/hmg/ddp505.

[9] Z. Erasmus, Apartheid race categories: daring to question their continued use, Transformation: Critical Perspectives on Southern Africa. 79 (2012) 1-11. doi:10.1353/trn.2012.0008.

[10] Statistics South Africa, Census 2011: Statistical Release, Statistics South Africa, Pretoria, 2012.

[11] D.C. Petersen, O. Libiger, E.A. Tindall, R.-A. Hardie, L.I. Hannick, R.H. Glashoff, et al., Complex Patterns of Genomic Admixture within Southern Africa, PLoS Genet. 9 (2013) e1003309. doi:10.1371/journal.pgen.1003309.

[12] R.E. Van der Ross, Up from slavery slaves at the Cape: their origins, treatment and contribution, Ampersand Press in association with the University of the Western Cape, Kenilworth [South Africa], 2005.

[13] C.K. Jacobson, A.Y. Amoateng, T.B. Heaton, Inter-Racial Marriages in South Africa*, Journal of Comparative Family Studies. 35 (2004) 443.

[14] I.D. MacCrone, Race attitudes in South Africa historical, experimental and psychological studies., 1st ed., Oxford University Press, London, 1937.

[15] R. Sherman, M. Steyn, E-race-ing the line: South African interracial relationships yesterday and today, in: The Prize and the Price: Shaping Sexualities in South Africa, HSRC Press, Cape Town, South Africa, 2009: pp. 55-81.

[16] C. Schlebusch, Issues raised by use of ethnic-group names in genome study, Nature. 464 (2010) 487-487. doi:10.1038/464487a.

[17] C.M. Schlebusch, P. Skoglund, P. Sjödin, L.M. Gattepaille, D. Hernandez, F. Jay, et al., Genomic Variation in Seven Khoe-San Groups Reveals Adaptation and Complex African History, Science. 338 (2012) 374-379. doi:10.1126/science. 1227721.

[18] C. Barbieri, A. Butthof, K. Bostoen, B. Pakendorf, Genetic perspectives on the origin of clicks in Bantu languages from southwestern Zambia, Eur. J. Hum. Genet. 21 (2013) 430-436. doi:10.1038/ejhg.2012.192.

[19] M. Henneberg, G. Brush, G.A. Harrison, Growth of specific muscle strength between 6 and 18 years in contrasting socioeconomic conditions, American Journal of Physical Anthropology. 115 (2001) 62-70. doi:10.1002/ajpa.1057. 
[20] S.A. Tishkoff, F.A. Reed, F.R. Friedlaender, C. Ehret, A. Ranciaro, A. Froment, et al., The genetic structure and history of Africans and African Americans, Science. 324 (2009) 1035-1044. doi:10.1126/science.1172257.

[21] J.K. Pritchard, M. Stephens, P. Donnelly, Inference of population structure using multilocus genotype data, Genetics. 155 (2000) 945-959.

[22] L. Quintana-Murci, C. Harmant, H. Quach, O. Balanovsky, V. Zaporozhchenko, C. Bormans, et al., Strong Maternal Khoisan Contribution to the South African Coloured Population: A Case of Gender-Biased Admixture, Am J Hum Genet. 86 (2010) 611-620. doi:10.1016/j.ajhg.2010.02.014.

[23] J.L. McDowell, E.N. L'Abbé, M.W. Kenyhercz, Nasal aperture shape evaluation between black and white South Africans, Forensic Science International. 222 (2012) 397.e1-397.e6. doi:10.1016/j.forsciint.2012.06.007.

[24] E.N. L'Abbé, M. Kenyhercz, K.E. Stull, N. Keough, S. Nawrocki, Application of FORDISC 3.0 to explore differences among crania of North American and South African blacks and whites, J. Forensic Sci. 58 (2013) 1579-1583. doi:10.1111/15564029.12198.

[25] E.N. L'Abbé, C. Van Rooyen, S.P. Nawrocki, P.J. Becker, An evaluation of nonmetric cranial traits used to estimate ancestry in a South African sample, Forensic Science International. 209 (2011) 195.e1-195.e7. doi:10.1016/j.forsciint.2011.04.002.

[26] J.T. Hefner, Cranial Nonmetric Variation and Estimating Ancestry*, Journal of Forensic Sciences. 54 (2009) 985-995. doi:10.1111/j.1556-4029.2009.01118.x.

[27] D. Smay, G.J. Armelagos, Galileo wept: A critical assessment of the use of race in forensic anthropology, Transforming Anthropology. 9 (2000) 19-29.

[28] G.J. Armelagos, A.H. Goodman, Race, Racism, and Anthropology, in: A.H. Goodman, T.L. Leatherman (Eds.), Building a New Biocultural Synthesis, The University of Michigan Press, Ann Arbor, 1998.

[29] L.W. Konigsberg, S.D. Ousley, Multivariate quantitative genetics of anthropometric traits from the Boas data, Human Biology. 67 (1995) 481+.

[30] C.S. Sparks, R.L. Jantz, A reassessment of human cranial plasticity: Boas revisited, Proceedings of the National Academy of Sciences of the United States of America. 99 (2002) 14636-14639.

[31] S. Ousley, 3Skull, 2004.

[32] C. Klingenberg, MorphoJ: An integrated software package for geometric morphometrics., Moelcular Eco Res. 11 (2011) 353-357.

[33] F. Bookstein, Morphometric Tools for Landmark Data: Geometry and Biology, Cambridge University Press, Cambridge, 1991.

[34] I.T. Jolliffe, Principal Component Analysis, 2nd ed., Springer, New York, 2002.

[35] C. Klingenberg, Visualizations in geometric morphometrics: how to read and how to make graphs showing shape changes., Hystrix. 24 (2013) 15-24.

[36] C. Huberty, Applied Discriminant Analysis, Wiley and Sons, New York, 1994.

[37] B.G. Tabachnick, L.S. Fidell, Using multivariate statistics, 5th ed., Pearson Education, Boston, 2007.

[38] J.H. Relethford, H.C. Harpending, Craniometric variation, genetic theory, and modern human origins, Am. J. Phys. Anthropol. 95 (1994) 249-270. doi:10.1002/ajpa.1330950302. 
[39] J.H. Relethford, Craniometric variation among modern human populations, American Journal of Physical Anthropology. 95 (1994) 53-62.

[40] J.H. Relethford, Boas and beyond: Migration and craniometric variation, AMerican Journal of Human Biology. 16 (2004) 379-386.

[41] K. Harvati, T.D. Weaver, Human cranial anatomy and the differential preservation of population history and climate signatures, Anat Rec A Discov Mol Cell Evol Biol. 288 (2006) 1225-1233. doi:10.1002/ar.a.20395.

[42] H. Tang, T. Quertermous, B. Rodriguez, S.L.R. Kardia, X. Zhu, A. Brown, et al., Genetic Structure, Self-Identified Race/Ethnicity, and Confounding in Case-Control Association Studies, Am J Hum Genet. 76 (2005) 268-275.

[43] A.H. McKeown, D. Wescott, Sex and Ancestry Estimation from Landmarks of the Cranial Base, in: American Academy of Forensic Sciences Annual Scientific Meeting, Seattle, 2010.

[44] S. Dubow, Scientific Racism in Modern South Africa, Cambrige University Press, Cambridge, 1995.

[45] M. Hall, A. Morris, Race and Iron age human skeletal remains from Southern Africa: An assessment, Social Dynamics. 9 (1983) 29-36.

doi:10.1080/02533958308458344. 